\title{
JAK2 V617F-Mutated Myeloproliferative Neoplasia Developing Five Years after Wild-Type JAK2 Acute Myeloid Leukemia: A Case Report
}

\author{
Sabine Girsberger ${ }^{a} \quad$ Axel Karow $^{b}$ Pontus Lundberg ${ }^{b}$ Stephan Dirnhofer ${ }^{c}$ \\ Thomas Lehmann $^{\mathrm{a}}$ Jakob R. Passweg ${ }^{\mathrm{a}}$ André Tichelli $^{\mathrm{a}}$ Radek Skoda $^{\mathrm{b}}$ \\ Alicia Rovó ${ }^{a}$ \\ ${ }^{a}$ Department of Hematology, ${ }^{b}$ Department of Biomedicine, Experimental Hematology and ${ }^{\mathrm{C}}$ Institute of Pathology, \\ University Hospital Basel, Basel, Switzerland
}

Myeloproliferative neoplasms (MPN) are clonal stem cell disorders characterized by increased proliferation of one to three hematopoietic cell lineages. The protein tyrosine kinase JAK2 is mutated in virtually all patients with polycythemia vera and approximately $60 \%$ of patients with primary myelofibrosis or essential thrombocythemia [1]. In the past years less prevalent mutations involving MPL, TET2, ASXL1, CBL, IDH1 and IDH2, EZH2, DNMT3A as well as IKZF1 have additionally been described [2]. The transformation of MPN in acute myeloid leukemia (AML) is a well-reported phenomenon. However, how JAK2 and other MPN-associated mutations contribute to disease progression is still the subject of debate [2-4]. In contrast, JAK2-positive MPN as secondary neoplasia occurring after AML is a rare clinical observation with only few case reports in the literature [5, 6] and little is known about the underlying pathophysiology. Here we report the unique case of a 62 -year-old patient with secondary MPN harboring JAK2 V617F, which presented with isolated thrombocytosis 4 years after successful treatment with chemotherapy of a JAK2-negative AML. The idea that the expansion of the JAK2 V617F clone is associated with AML-linked factors is supported by the fact that our patient had a third malignancy, pancreatic cancer, associated with dysregulation in JAK/ STAT3 signaling.

\section{KARGER \\ Fax +4161306 1234 \\ E-Mail karger@karger.ch}

www.karger.com
(C) 2012 S. Karger AG, Basel

0001-5792/13/1291-0023\$38.00/0

Accessible online at:

www.karger.com/aha
In March 2006 a 57-year-old male was admitted to hospital, complaining of a short period of asthenia and night sweats. Laboratory analysis demonstrated markedly elevated leukocytes $\left(37 \times 10^{9} / 1\right)$ with $87 \%$ of blasts, hemoglobin of $10.4 \mathrm{~g} / \mathrm{dl}$ and thrombocytopenia of $43 \times 10^{9} / 1$. Subsequent bone marrow investigation revealed dominant leukemic infiltration (90\%) composed of myeloid blasts without maturation, consistent with the diagnosis of AML not otherwise categorized (according to WHO 2008), morphologically type AML without maturation. Cytogenetics as well as molecular analysis remained unremarkable, giving no evidence of recurrent genetic abnormalities. The patient was treated on HOVON 42 Protocol Arm A receiving 2 cycles of induction chemotherapy with cytarabine/idarubicin and cytarabine/amsacrine, respectively, which resulted in early first complete remission. According to the protocol and due to the lack of an appropriate donor for allogeneic hematopoietic stem cell transplantation, therapy was completed with consolidation chemotherapy with mitoxantrone/etoposide.

Four years later, in June 2010, a slight thrombocytosis was first noted and increased in time to $717 \times 10^{9} / 1$ in June 2011, with otherwise normal full blood count values. Peripheral blood analysis for the JAK2 V617F mutation was positive (allele burden 34\%). The red cell mass as well as the EPO level was in the normal range, and bone mar-

Dr. Sabine Girsberger

University Hospital Basel, Hematology

Petersgraben 4

$\mathrm{CH}-4031$ Basel (Switzerland)

E-Mail girsbergers@uhbs.ch 


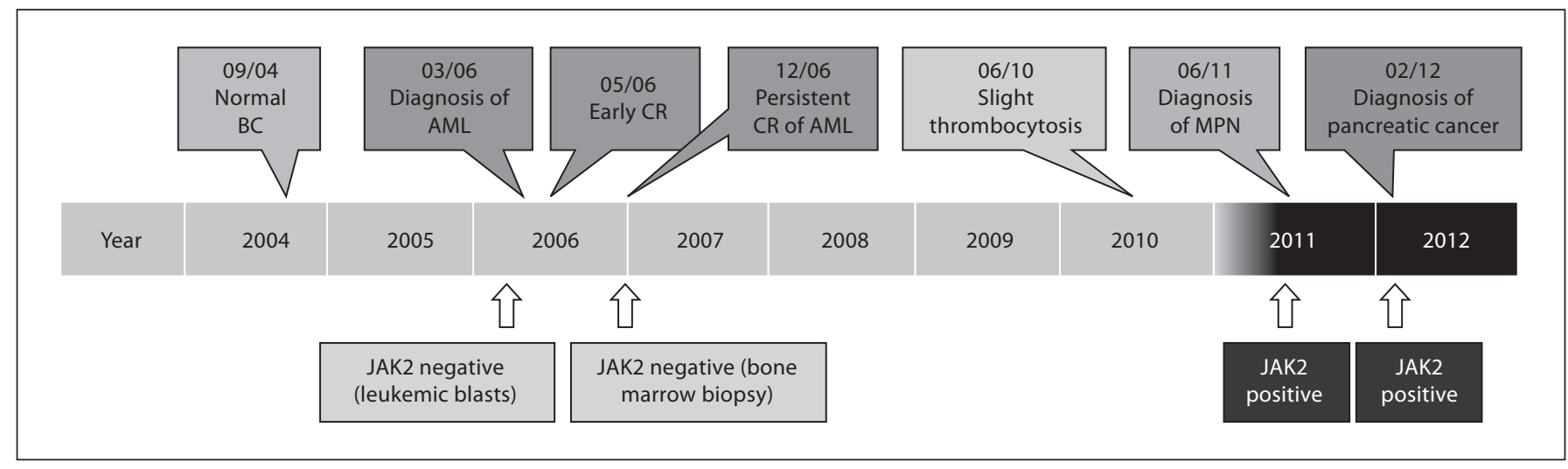

Fig. 1. Sequence of disease and JAK2 V617F mutational status. BC = Blood count; $\mathrm{CR}=$ complete remission.

row cytology and biopsy revealed signs of myeloproliferation without evidence of relapsing acute leukemia. The diagnosis of myeloproliferative neoplasia, most likely consistent with essential thrombocythemia, was established according to WHO 2008 criteria [7]. We retrospectively assessed JAK2 V617F mutational status in cryopreserved leukemic blasts and bone marrow biopsies at the diagnosis of AML and at remission time and all tests proved negative for the V617F mutant allele. Additional sequencing of genes frequently mutated in MPN or AML (MPL, TET2, ASXL1, CBL, IDH1 and IDH2, TP53, FLT3 and RUNX1) did not disclose any other somatic mutation, neither at AML diagnosis nor at MPN. The blood cell counts prior to AML diagnosis were unremarkable, excluding a preexisting, unrecognized, transformed MPN.

Cytoreductive treatment with hydroxyurea was initiated and led to normalization of platelet count during follow-up. In February 2012, the patient presented with severe abdominal pain and was diagnosed as having pancreatic cancer with peritoneal carcinosis (fig. 1). JAK2 V617F mutation on extracted DNA from pancreatic tumor cells turned out to be positive (allele burden 6\%).

The findings of our case report were considered conclusive for the diagnosis of a secondary JAK2-positive MPN occurring after treatment of wild-type AML. The uncommon sequence of the disease presentation raises a number of questions concerning the pathogenesis of both hematological diseases as well as the pancreatic tumor. It is suggestive that the expansion of the JAK2 V617F clone is associated with AML-linked factors. It is possible that direct or indirect mechanisms led to JAK2 mutation through either predisposing factors in the initial AML clone or induced by chemotherapy. The idea of an AML-linked signaling alter- ation is supported by the fact that our patient had a second malignancy, pancreatic cancer, which has been described in the literature to be associated with dysregulation of JAK/ STAT3 signaling [8]. In contrast to a former study where STAT3 activation in pancreatic cancer was postulated to be driven by another mechanism than JAK2 V617F mutation [9] the pancreatic tumor cells in our case turned out to harbor the JAK2 V617F mutation. However, in the case of a JAK2 V617F-positive disease-initiating pancreatic tumor cell, an allele burden of close to $100 \%$ would be expected. Considering the low allele burden of only $6 \%$ in our case, a contamination by intratumoral blood cells is very likely.

On the other hand, both hematological diseases might be the expression of different phenotypes of the same clone, not fully eradicated by chemotherapy. Regarding our case, the lack of additional somatic mutations could neither support nor exclude this hypothesis. Furthermore, the idea of a favorable environment for the expansion of a preexisting JAK2 V617F clone [5] is not contradictory to our case since the JAK2 V617F clone could have been below detection limit at AML diagnosis. Finally, the two hematological diseases could represent transformation of two completely independent stem cell clones. In agreement with the previously published case reports, the time interval of 4 to 5 years between chemotherapy and clinical evidence of MPN is similar.

In conclusion, this unique case suggests an association between the initial AML and the following JAK2-positive secondary MPN as well as pancreatic cancer. Closer understanding of this complex phenomenon, especially the role of the AML clone itself and the effect of chemotherapy on stem cell function and generation of JAK2 and other mutations, needs further investigation. 


\section{References}

$>1$ Vannucchi AM, Guglielmelli P, Tefferi A: Advances in understanding and management of myeloproliferative neoplasms. CA Cancer J Clin 2009;59:171-191.

$>2$ Tefferi A: Novel mutations and their functional and clinical relevance in myeloproliferative neoplasms: JAK2, MPL, TET2, ASXL1, CBL, IDH and IKZF1. Leukemia 2010;24:1128-1138.

3 Theocharides A, Boissinot M, Girodon F, Garand R, Teo SS, Lippert E, Talmant P, Tichelli A, Hermouet S, Skoda RC: Leukemic blasts in transformed JAK2-V617F-positive myeloproliferative disorders are frequently negative for the JAK2-V617F mutation. Blood 2007;110:375-379.
4 Klampfl T, Harutyunyan A, Berg T, Gisslinger B, Schalling M, Bagienski K, Olcaydu D, Passamonti F, Rumi E, Pietra D, Jäger R, Pieri L, Guglielmelli P, Iacobucci I, Martinelli G, Cazzola M, Vannucchi AM, Gisslinger H, Kralovics R: Genome integrity of myeloproliferative neoplasms in chronic phase and during disease progression. Blood 2011; 118:167-176.

5 Portell CA, Sekeres MA, Rogers HJ, Tiu RV: De novo polycythaemia vera arising 5 years following acute myeloid leukemia remission: suggestion of a chemotherapy resistant JAK2 clone. Br J Haematol 2012;157:266-267.

6 Belotti A, Doni E, Rossi V, Pioltelli P, Pogliani EM: Development of polycythemia vera after chemotherapy-induced remission of acute myeloid leukemia: a case report. Acta Haematol 2011;126:52-53.
7 Tefferi A, Vardiman JW: Classification and diagnosis of myeloproliferative neoplasms: the 2008 World Health Organization criteria and point-of-care diagnostic algorithms. Leukemia 2008;22:14-22.

8 Huang C, Cao J, Huang KJ, Zhang F, Jiang T, Zhu L, Qiu ZJ: Inhibition of STAT3 activity with AG490 decreases the invasion of human pancreatic cancer cells in vitro. Cancer Sci 2006;97:1417-1423.

9 Kocher HM, Mears L, Lea NC, Raj K, Mufti GJ: JAK V617F missense mutation is absent in pancreatic cancer. Gut 2007;56:1174-1175. 